\title{
Projectivity of the Space of Divisors on a Normal Compact Complex Space
}

By

\author{
Akira FUJIKI*
}

\section{Introduction}

For any complex space we shall denote by $D_{X}$ the Douady space of compact complex subspaces of $X$ [1]. Let $Z_{X} \subseteq D_{X} \times X$ be the universal subspace so that for each $d \in D_{X}$, the corresponding subspace of $X$ is given by $Z_{X, d}:=Z_{X} \cap(\{d\} \times X)$ $\subseteq\{d\} \times X=X$. Recall that a Cartier divisor on $X$ is a complex subspace of $X$ whose sheaf of ideals is generated locally by a single element which is not a zero divisor. Let $\operatorname{Div} X=\left\{d \in D_{X} ; Z_{X, d}\right.$ is a Cartier divisor on $\left.X\right\}$. Then $\operatorname{Div} X$ is Zariski open in $D_{X}$, and in fact is a union of connected components of $D_{X}$ when $X$ is nonsingular. Then the purpose of this paper is to prove the following:

Theorem $\mathbb{1}$. For any normal compact complex space $X$ every connected component of $\operatorname{Div} X$ is compact and projective.

When $X$ is nonsingular, the proof actually gives a more precise structure theorem of Div $X$ (cf. Proposition in $\S 1$ below). The motivation for this theorem comes from Fischer-Forster [2] where they proved that there exist only a finite number of reduced divisors on any compact complex manifold $X$ which are mapped surjectively onto $Y$ where $f: X \rightarrow Y$ is an algebraic reduction of $X$ (cf. $\S 1$ ); this implies that almost all the divisors on $X$ are obtained as the pull-backs of those on $Y$ which is projective. Theorem 1 reveals a striking contrast to the case of codimension $>1$, where in order to obtain the compactness even of the irreducible components of $D_{X}$ in general, it is necessary to assume that $X$ is Kähler or more generally that $X$ is in $\mathscr{C}$ (cf. [9]). Indeed, the analogy of [2] fails in codimension $>1$ as was shown by Campana [0].

Though we prove the compactness and projectivity at the same time in Theorem 1 , there is an easy alternative proof for the projectivity once the com-

Commuincated by S. Nakano, January 21, 1982.

* Department of Mathematics, Yoshida College, Kyoto University, Kyoto 606, Japan. 
pactness is established. Namely we shall also show the following theorem, stimulated by a result of Ohsawa ([8] Theorem 2) (cf. Remark 2).

Theorem 2. Let $X$ be a connected normal compact complex space and $A$ an analytic subset of $X$. Then for any irreducible component $D_{\alpha}$ of $\operatorname{Div} X$ the subset $D_{\alpha}(A):=\left\{d \in D_{\alpha} ; Z_{X, d} \cap A \neq \emptyset\right\}$ is a support of an ample divisor on $D_{\alpha}$ if $D_{\alpha}(A) \neq D_{\alpha}$.

The projectivity is used in [3] to get a local projectivity of a model of a relative algebraic reduction for a fiber space in $\mathscr{C}$.

Convention. For any complex space $B, B_{\text {red }}$ denotes the underlying reduced subspace. A complex variety is a reduced and irreducible complex space. A morphism $f: X \rightarrow Y$ of complex varieties is called a fiber space if $f$ is proper and the general fiber of $f$ is irreducible.

\section{§1. Preliminary Reductions}

Let $f: X \rightarrow Y$ be a morphism of complex varieties. Let $Z \cong X$ be a Cartier divisor on $X$. Then we call $Z$ a relative divisor over $Y$ if the following equivalent conditions are satisfied: 1) $Z$ is flat over $Y$. 2) $Z$ contains no irreducible component of the fibers of $f$ (cf. [5], 21.15).

Conversely, if $Z \subseteq X$ is a subspace which is flat over $Y$ and if $Z_{y}$ is a Cartier divisor on $X_{y}$ for every $y \in Y$, then $Z$ is a relative divisor over $Y([5])$.

Thus if we set $Z(X)=Z_{X} \cap(\operatorname{Div} X \times X) \subseteq \operatorname{Div} X \times X, Z(X)$ is a relative divisor over $\operatorname{Div} X, Z_{X}$ being flat over $D_{X}$. Further $\operatorname{Div} X$ has the following universal property. Let $Z \subseteq T \times X$ be a relative divisor over $T$ with respect to the natural morphism $\rho_{T}: Z \rightarrow T$ where $T$ is any complex space. Then there exists a unique morphism $\tau: T \rightarrow \operatorname{Div} X$ such that $Z=\left(\tau \times i d_{X}\right)^{-1}(Z(X))$, and hence, that $\rho_{T}$ is induced from the universal morphism $\rho: Z(X) \rightarrow \operatorname{Div} X$. So we shall call $Z(X)$ the universal divisor associated to $X$.

Let $X$ be a compact complex space. Let $\operatorname{Pic} X=H^{1}\left(X, \mathcal{O}_{X}^{*}\right)$ be the Picard variety of $X$, which has the natural structure of a commutative complex Lie group [6]. Let $c_{1}: H^{1}\left(X, \mathcal{O}_{X}^{*}\right) \rightarrow H^{2}(X, \mathbb{Z})$ be the first chern class map and $N S(X)$ $=\operatorname{Im} c_{1}$, the Nerson-Severi group of $X$. For $\gamma \in N S(X)$ we set $\operatorname{Pic}_{\gamma} X=c_{1}^{-1}(\gamma)$ so that we have

$$
\operatorname{Pic} X=\coprod_{\gamma \in N S(X)} \operatorname{Pic}_{\gamma} X
$$


In particular $\operatorname{Pic}_{0} X=\operatorname{Ker} c_{1}$ is the identity component of Pic $X$.

Now $Z(X)$ defines a line bundle $[Z(X)]$ on $\operatorname{Div} X \times X$ and then by the universality of $\operatorname{Pic} X$ we have the natural morphism $\mu_{X}$ : Div $X \rightarrow \operatorname{Pic} X$, which eventually associates to each $d \in \operatorname{Div} X$ the corresponding line bundle $\left[Z(X)_{d}\right]$ (cf. [3] and [4], exposé 234, §4). Further, we know that $\mu_{X}$ is projective and the fiber over $L \in \operatorname{Pic} X$ is naturally identified with the projective space $\mathbb{P}(\Gamma(X, L))$ : $=(\Gamma(X, L)-\{0\}) / \mathbb{C}^{*}$, i.e., the linear system on $X$ associated to $L$, if it is not empty (cf. [3], [4]). Thus if we set $(\operatorname{Div} X)^{-}:=\mu_{X}(\operatorname{Div} X) \subseteq \operatorname{Pic} X$, the following lemma holds.

Lemma 1. $(\operatorname{Div} X)_{\text {red }}^{-}=\left\{p \in \operatorname{Pic} X ; \operatorname{dim} \Gamma\left(X, L_{p}\right)>0\right\}-\{e\}$ where $e$ is the identity of $\operatorname{Pic} X$ and $L_{p}$ is the line bundle on $X$ corresponding to $p$.

Note that $(\operatorname{Div} X)^{-}$is an analytic subspace of $\operatorname{Pic} X$ as $\mu_{X}$ is proper, though this also follows from Lemma 1 easily. We set $D_{\gamma}=\mu_{X}^{-1}\left(\operatorname{Pic}_{\gamma} X\right)$ and $\mu_{\gamma}=\mu_{X} \mid D_{\gamma}$ : $D_{\gamma} \rightarrow \operatorname{Pic}_{\gamma} X$. Let $Z_{\gamma} \subseteq D_{\gamma} \times X$ be the restriction of $Z(X)$ over to $D_{\gamma}$. We have of course Div $X=\coprod_{\gamma} D_{\gamma}$. Let $\bar{D}_{\gamma}=\mu_{\gamma}\left(D_{\gamma}\right)$, so that $(\operatorname{Div} X)^{-}=\coprod_{\gamma} \bar{D}_{\gamma} . \quad$ Then by virtue of the above description of $\operatorname{Div} X$ we see that our task is to show that every connected component of $\bar{D}_{\gamma}$ is projective.

Let $X$ be a compact connected complex manifold. Let

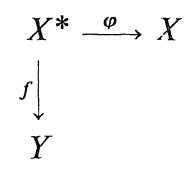

be a holomorphic model of algebraic reduction of $X$. Namely $X^{*}$ is a compact complex manifold, $\varphi$ is a bimeromorphic morphism, $Y$ is a projective manifold and $f$ is a fiber space which induces an isomorphism $f^{*}: \mathbb{C}(Y) \cong \mathbb{C}\left(X^{*}\right)$ of the meromorphic function fields of $X^{*}$ and $Y$. Let $f^{*}: \operatorname{Pic}_{0} Y \rightarrow \operatorname{Pic}_{0} X^{*}$ and $\varphi^{*}$ : $\operatorname{Pic}_{0} X \rightarrow \mathrm{Pic}_{0} X^{*}$ be the natural homomorphisms. Then we know that $\varphi^{*}$ is isomorphic and $f^{*}$ is injective (since $f_{*} \mathcal{O}_{X^{*}} \cong \mathcal{O}_{Y}$ ). Thus we get an injective homomorphism $\varphi^{*-1} f^{*}: \operatorname{Pic}_{0} Y \rightarrow \operatorname{Pic}_{0} X$. We omit the proof of the following lemma, which is standard.

Lemma 2. The abelian (group) subvariety $P^{a}$ of $\mathrm{Pic}_{0} X$, which is by definition the image of $\operatorname{Pic}_{0} Y$ via $\varphi^{*-1} f^{*}$, is independent of the choice of a holomorphic model of algebraic reduction of $X$ as above and depends only on $X$.

Each $\operatorname{Pic}_{\gamma} X$ is naturally a principal homogeneous space under $\operatorname{Pic}_{0} X$. 
We denote by $P_{\gamma}^{a} \subseteq \operatorname{Pic}_{\gamma} X$ any orbit of the induced action of $P^{a} \subseteq \operatorname{Pic}_{0} X$ on $\operatorname{Pic}_{\gamma} X$.

Lemma 3. Let $\gamma \in N S(X)$. Let $C$ be any complex subspace of $\operatorname{Pic}_{\gamma} X$ such that $C_{\mathrm{red}}$ is contained in an orbit $P_{\gamma}^{a}$ of $P^{a}$ in $\mathrm{Pic}_{\gamma} X$. Then $C$ is projective.

Proof. Clearly we may assume that $\operatorname{Pic}_{\gamma} X=\operatorname{Pic}_{0} X$ and $P_{\gamma}^{a}=P^{a}$. Let $\bar{P}^{a}=\left(\operatorname{Pic}_{0} X\right) / P^{a}$ be the quotient Lie group and $q: \mathrm{Pic}_{0} X \rightarrow \bar{P}_{a}$ the natural homomorphism. Let $\bar{e}=q(e)$. Since $q$ is a holomorphic fiber bundle with typical fiber $P^{a}$ there exists a neighborhood $V$ of $\bar{e}$ such that $q$ is projective (in fact trivial) over $V$. In particular any infinitesimal neighborhood of $P^{a}$ in $\operatorname{Pic}_{0} X$, e.g., the space $P_{(n)}^{a}=\left(P^{a}, \mathcal{O}_{\mathrm{Pic}_{0} X} / \mathscr{I}^{n+1}\right)$ where $\mathscr{I}$ is the defining ideal of $P^{a}$ in $\mathrm{Pic}_{0} X$, is projective. Since $C \subseteq P_{(n)}^{a}$ for some $n>0$ by our assumption, $C$ also is projective.

q.e.d.

By this lemma, if we prove the next proposition, Theorem would follow in the case $X$ is connected and nonsingular, in view of the projectivity of $\mu_{\gamma}$.

Proposition. Let $X$ be a compact complex manifold. Let $\gamma \in N S(X)$. Then any connected component of $\bar{D}_{\gamma, \mathrm{red}}:=\mu_{\gamma}\left(D_{\gamma, \mathrm{red}}\right)$ is contained in an orbit $P_{\gamma}^{a}$ of $P^{a}$ on $\operatorname{Pic}_{\gamma} X$.

\section{§2. Proof of Proposition}

First we shall fix some notations. Let $X$ be a complex space.

Let $n$ : Div $X \rightarrow(\operatorname{Div} X)_{\text {red }}$ be the normalization of $(\operatorname{Div} X)_{\text {red }}$ and $\tilde{\rho}$ : $\tilde{Z}(X) \rightarrow \tilde{D} i v X$ be the pull-back of the universal family to Div $X$. Let $T$ be any normal complex space and $Z \cong T \times X$ a relative divisor over $T$. Then by the universal property of $\operatorname{Div} X$ and the normality of $T$ we can find a morphism $\tau$ : $T \rightarrow \tilde{D}$ iv $X$ (not necessarily unique) such that $\rho_{T}: Z \rightarrow T$ is induced from $\tilde{\rho}$ via $\tau$. We call any such morphism also a universal map associated to $\rho_{T}$. For any irreducible component $D_{\alpha}$ of (Div $\left.X\right)_{\text {red }}$ we denote by $\tilde{D}_{\alpha}$ the corresponding irreducible component of Div $X$ and by $\tilde{Z}_{\alpha} \rightarrow \tilde{D}_{\alpha}, \tilde{Z}_{\alpha} \subseteq \widetilde{D}_{\alpha} \times X$, the pull-back of the universal family to $\widetilde{D}_{\alpha}$. Then $n_{\alpha}:=\left.n\right|_{\tilde{D}_{\alpha}}: \widetilde{D}_{\alpha} \rightarrow D_{\alpha}$ is the normalization of $D_{\alpha}$.

Let 'Div $X$ (resp. 'D̃iv $X$ ) be the union of those irreducible components $D_{\alpha}$ of $(\operatorname{Div} X)_{\text {red }}$ (resp. $\tilde{D}_{\alpha}$ of D̃iv $X$ ) such that $Z_{\alpha}$ (resp. $\tilde{Z}_{\alpha}$ ) is reduced and irreducible. Then $n$ induces $n^{\prime}:$ 'Div $X \rightarrow{ }^{\prime} \operatorname{Div} X$ which is the normalization of 'Div $X$.

Let $Z \subseteq T \times X$ and $\tau: T \rightarrow \tilde{D}$ iv $X$ be as above with $Z$ reduced and irreducible. 
If $\tilde{D}_{\alpha}$ is the irreducible component containing $\tau(T)$, then $\tilde{D}_{\alpha} \subseteq \tilde{D}^{\prime} \mathrm{iv} X$. In fact, if $\tilde{Z}_{\alpha}$ is either nonreduced or irreducible, then $\tilde{Z}_{\alpha} \times_{\widetilde{D}_{\alpha}} T$ is either nonreduced or irreducible.

We record the following useful result of C. P. Ramanujam.

Lemma 4. Let $X$ be a complex manifold and $S$ a normal complex space. Let $Z \subseteq S \times X$ be a reduced analytic subspace of pure codimension 1 . Suppose that $Z$ contains no subspace of the form $\{s\} \times X, s \in S$. Then $Z$ is a relative divisor over $S$.

Proof. See [5], 21.14.1.

The next lemma reduces our problem to considering 'D̃iv $X$.

Lemma 5. Let $X$ be a compact complex manifold. Let $D_{\alpha}$ be any irreducible component of $(\operatorname{Div} X)_{\mathrm{red}}$. Then there exist irreducible components $D_{\alpha_{1}}, \ldots, D_{\alpha_{m}}$ of $(\operatorname{Div} X)_{\mathrm{red}}$ and an isomorphism $\varphi_{\alpha}: \tilde{D}_{\alpha_{1}} \times \cdots \times \tilde{D}_{\alpha_{m}} \rightarrow \tilde{D}_{\alpha}$ such that 1) $\widetilde{Z}_{\alpha_{i}}$ are reduced and irreducible, i.e., $\widetilde{D}_{\alpha_{i}} \subseteq$ 'Div $X$ and 2) if $\mu_{X}\left(D_{\alpha}\right) \subseteq \operatorname{Pic}_{\gamma} X$ and $\mu_{X}\left(D_{\alpha_{i}}\right) \subseteq \operatorname{Pic}_{\gamma_{i}} X$, then $\gamma=\gamma_{1}+\cdots+\gamma_{m}$ and $\tilde{\mu}_{\alpha} \varphi_{\alpha}=\psi_{\alpha}\left(\tilde{\mu}_{\alpha_{1}} \times \cdots \times \tilde{\mu}_{\alpha_{m}}\right)$ where $\tilde{\mu}_{\alpha}=\mu_{X} n_{\alpha}, \tilde{\mu}_{\alpha_{i}}=\mu_{X} n_{\alpha_{i}}$ and $\psi_{\alpha}: \operatorname{Pic}_{\gamma_{1}} X \times \cdots \times \operatorname{Pic}_{\gamma_{m}} X \rightarrow \operatorname{Pic}_{\gamma} X$ is given by $\psi_{\alpha}\left(p_{1}, \ldots\right.$, $\left.p_{m}\right)=p_{1}+\cdots+p_{m}($ addition in Pic $X)$.

Proof. Let $\widetilde{Z}_{\alpha, i} \subseteq \widetilde{D}_{\alpha} \times X, i=1, \ldots, m$, be the irreducible components of $\tilde{Z}_{\alpha, \text { red }}$ and $\mathscr{J}_{i}$ their ideal sheaves. Since $\tilde{Z}_{\alpha}$ is a relative divisor over $\tilde{D}_{\alpha}$ and $\tilde{D}_{\alpha}$ is normal, by Lemma $4 \tilde{Z}_{\alpha, i}$ are also relative divisors over $\tilde{D}_{\alpha}$. Moreover $\mathscr{J}$ $=\mathscr{J}_{1}^{k_{1}} \ldots \mathscr{J}_{m}^{k_{m}}$ is the ideal sheaf of $\tilde{Z}_{\alpha}$ for unique positive integers $k_{i}$ (cf. [5] IV, 21.6.9). Let $\tau_{i}: \widetilde{D}_{\alpha} \rightarrow \tilde{D}$ iv $X$ be a universal morphism associated to $\tilde{Z}_{\alpha, i} \rightarrow \tilde{D}_{\alpha}$. Let $\widetilde{D}_{\alpha}$ be the irreducible component of Div $X$ which contains $\tau_{i}\left(\widetilde{D}_{\alpha}\right)$. Let $\hat{D}_{\alpha}$ $=\tilde{D}_{\alpha_{1}} \times \cdots \times \tilde{D}_{\alpha_{m}}$. Let $\hat{Z}_{\alpha_{i}}:=\tilde{D}_{\alpha_{1}} \times \cdots \times \tilde{Z}_{\alpha_{i}} \times \cdots \times \tilde{D}_{\alpha_{m}} \quad\left(\tilde{Z}_{\alpha_{i}}\right.$ on the $i$-th place $)$ naturally considered as a subspace of $\hat{D}_{\alpha} \times X$. Let $\mathscr{J}_{i}^{\prime}$ be the ideal sheaf of $\hat{Z}_{\alpha_{i}}$. Let $\widehat{Z}_{\alpha} \subseteq \widehat{D}_{\alpha} \times X$ be the relative divisor defined by the ideal sheaf $\mathscr{J}^{\prime}=\mathscr{J}_{1}^{\prime k_{1}} \ldots \mathscr{J}_{m}^{\prime k_{m}}$ (cf. Lemma 4). Let $\varphi_{\alpha}: \widehat{D}_{\alpha} \rightarrow$ D̃iv $X$ be an associated universal morphism. From our construction it then follows readily that $\varphi_{\alpha}\left(\tau_{1} \times \cdots \times \tau_{m}\right)$ induces the identity of $\widetilde{D}_{\alpha}$. In particular $\widetilde{D}_{\alpha} \subseteq \varphi_{\alpha}\left(\hat{D}_{\alpha}\right)$. However, since $\hat{D}_{\alpha}$ is irreducible and $\tilde{D}_{\alpha}$ is an irreducible component of $\tilde{D}$ iv $X$ it follows that $\tilde{D}_{\alpha}=\varphi_{\alpha}\left(\hat{D}_{\alpha}\right)$. On the other hand, again from our construction it is clear that for any distinct points $d=\left(d_{1}, \ldots, d_{m}\right), d^{\prime}=\left(d_{1}^{\prime}, \ldots, d_{m}^{\prime}\right) \in \hat{D}_{\alpha}, \mathscr{J}_{d}^{\prime} \neq \mathscr{J}_{d^{\prime}}$. Hence $\varphi_{\alpha}$ is injective. Since both $\widetilde{D}_{\alpha}$ and $\hat{D}_{\alpha}$ are normal, this implies that $\varphi_{\alpha}$ is isomorphic. Moreover, since $\tilde{Z}_{\alpha, i}$ are reduced and irreducible, the same is true for $\widetilde{Z}_{\alpha i} ; 1$ ) follows. We show 2). 
Let $d=\left(d_{1}, \ldots, d_{m}\right) \in \hat{D}_{\alpha}$. Then from our construnction $\psi_{\alpha}\left(\tilde{\mu}_{\alpha_{1}} \times \cdots \times \tilde{\mu}_{\alpha_{m}}\right)(d)$ $=c_{1}\left(\left[\tilde{Z}_{\alpha_{1}, d_{1}}\right]^{k_{1}}\right)+\cdots+c_{1}\left(\left[\tilde{Z}_{\alpha_{k}, d_{k}}\right]^{k_{m}}\right)=c_{1}\left(\left[\tilde{Z}_{\alpha_{1}, d_{1}}\right]^{k_{1}} \otimes \cdots \otimes\left[\tilde{Z}_{\alpha_{k}, d_{k}}\right]^{k_{m}}=c_{1}\left(\left[\tilde{Z}_{\alpha, \varphi_{\alpha}(d)}\right]\right)\right.$ $=\tilde{\mu}_{\alpha} \varphi_{\alpha}(d)$.

Lemma 6. Let $f: X \rightarrow Y$ be a fiber space of compact complex varieties. Let $T$ be a complex variety and $Z \cong T \times X$ a relative divisor over $T$. Then the following conditions are equivalent. 1) $f\left(Z_{t}\right)=Y$ for all $t \in T$, and 2) $f\left(Z_{t}\right)$ $=Y$ for some $t \in T$.

Proof. Let $\bar{Z}=\left(i d_{T} \times f\right)(Z) \leqq T \times Y$. By the upper semi-continuity of dimensions of the fibers of $\bar{Z} \rightarrow T$ we see that the set $A=\left\{t \in T ; \bar{Z}_{t}=f\left(Z_{t}\right)=Y\right\}$ is analytic in $T$ where we identify $\{t\} \times X$ with $X$ and $\{t\} \times Y$ with $Y$. Let $r$ $=\operatorname{dim} X-\operatorname{dim} Y$. Let $B=\left\{(t, y) \in \bar{Z} ; \operatorname{dim} Z_{t, y} \geqq r\right\}$ where $Z_{t, y}=\{t\} \times \mathrm{f}_{\bar{Z}}^{-1}(y)$. By the same reason as above $B$ is analytic in $\bar{Z}$. Then for any $t \in A, B_{t}:=B \cap(\{t\}$ $\times Y) \neq \bar{Z}_{t}=\{t\} \times Y$; otherwise $\operatorname{dim} Z_{t}=\operatorname{dim} Y+r=\operatorname{dim} X$ so that $Z_{t}=X$. Hence if $A \neq \emptyset$, by the upper semi-continuity $\operatorname{dim} Z_{t, y}<r$ for general $(t, y) \in \bar{Z}$ and then for general $t \in T-A, \operatorname{dim} Z_{t}<\operatorname{dim} \bar{Z}_{t}+r<\operatorname{dim} Y+r=\operatorname{dim} X$, i.e., $\operatorname{dim} Z_{t} \leqq \operatorname{dim} X-2$. This is impossible. Thus either $A=\varnothing$ or $A=T$. This shows the equivalence of the lemma.

q.e.d.

Definition. Let $f: X \rightarrow Y$ be as in the above lemma. Let $\widetilde{D}_{\alpha}$ be any irreducible component of Div $X$. i) $\quad \tilde{D}_{\alpha}$ is called transversal to $f$ if $f\left(\tilde{Z}_{\alpha, d}\right)=Y$ for some $d \in \widetilde{D}_{\alpha}$ (and hence for all $d \in \widetilde{D}_{\alpha}$ by the above lemma). ii) $\widetilde{D}_{\alpha}$ is called isolated if $\tilde{D}_{\alpha}$ consists of a single point.

Remark 1. $\widetilde{D}_{\alpha}$ is isolated if and only if there exists a proper analytic subset $A \cong X$ such that the supports of $\tilde{Z}_{\alpha, d}$ are contained in $A$ for all $d \in \tilde{D}_{\alpha}$. In fact, since there exist at most countably many divisors whose supports are contained in $A, \widetilde{Z}_{\alpha} \rightarrow \widetilde{D}_{\alpha}$ is a trivial family in the sense that $\tilde{Z}_{\alpha, d}=\widetilde{Z}_{\alpha, d^{\prime}}$ for all $d, d^{\prime} \in D_{\alpha}$. More generally if $Z \leqq T \times X$ is a relative divisor over $T$ where $T$ is any connected complex space, and if $Z_{t} \leqq A$ for any $t \in T$ with $A$ as above then $Z \rightarrow T$ is a trivial family in the sense that $Z_{t}=Z_{t^{\prime}}$ for any $t, t^{\prime} \in T$. (The proof is the same.)

Lemma 7. Let $f: X \rightarrow Y$ be a fiber space of compact complex manifolds. Then there exists a natural bijective correspondence between the set $\mathfrak{E}_{X}$ of nonisolated and non-transversal irreducible components of ' $\tilde{D}_{\mathrm{iv}} X$ and the set $\mathbb{E}_{Y}$ of non-isolated irreducible components of 'Div $Y$ in such a way that if $\widetilde{D}_{\alpha} \in \mathfrak{E}_{X}$ and $\widetilde{D}_{\beta} \in \mathfrak{E}_{Y}$ correspond to each other, then there exist an isomorphism $\varphi_{\beta \alpha}: \widetilde{D}_{\beta} \rightarrow \widetilde{D}_{\alpha}$ and a point $d \in \operatorname{Div} X$ such that if $\bar{d}=\mu(d) \in \operatorname{Pic} X$, then $\tilde{\mu}_{\alpha} \varphi_{\alpha \beta}$ 
$=\bar{d}^{*} f^{*} \tilde{\mu}_{\beta}$ as a morphism $\tilde{D}_{\beta} \rightarrow \operatorname{Pic}_{\alpha} X$ where $\tilde{\mu}_{\alpha}=\mu_{X} n_{\alpha}, \tilde{\mu}_{\beta}=\mu_{Y} n_{\beta}, f^{*}:$ Pic $Y$ $\rightarrow$ Pic $X$ and $\bar{d}^{*}$ is the translation by $\bar{d}$.

Proof. Let $U \subseteq Y$ be a Zariski open subset over which $f$ is smooth. First we specify the correspondence. Let $\widetilde{D}_{\beta}$ be any non-isolated irreducible component of 'Div $Y$. Let $F_{\beta}:=i d_{\tilde{D}_{\beta}} \times f: \widetilde{D}_{\beta} \times X \rightarrow \widetilde{D}_{\beta} \times Y$. Let $\widetilde{E}_{\beta}:=F_{\beta}^{*} \widetilde{Z}_{\beta} \subseteq \widetilde{D}_{\beta} \times X$ be the pull-back of $\tilde{Z}_{\beta}$ to $\widetilde{D}_{\beta} \times X$ as a divisor. Since $\tilde{Z}_{\beta}$ is reduced and irreducible and $\left.F_{\beta}\right|_{\tilde{D}_{\beta} \times X_{U}}=i d_{\tilde{D}_{\beta}} \times f_{U}: \widetilde{D}_{\beta} \times X_{U} \rightarrow \widetilde{D}_{\beta} \times U$ is a smooth fiber space, $\widetilde{E}_{\beta}$ $\cap\left(\tilde{D}_{\beta} \times X_{U}\right)=F_{\beta}^{-1}\left(\tilde{Z}_{\beta} \cap\left(\tilde{D}_{\beta} \times U\right)\right)$ also is reduced and irreducible. Hence there exists a unique irreducible component $\widetilde{E}_{\beta 1}$ of $\widetilde{E}_{\beta, \text { red }}$ such that $F_{\beta}\left(\widetilde{E}_{\beta 1}\right)=\widetilde{Z}_{\beta}$. (Note that since $\widetilde{D}_{\beta}$ is non-isolated $\widetilde{E}_{\beta 1} \cap\left(\widetilde{D}_{\beta} \times X_{U}\right) \neq \varnothing$ by Remark 1.) Since $f$ is surjective, $\widetilde{E}_{\beta 1}$ contains no subspace of the form $\{d\} \times X, d \in \widetilde{D}_{\beta}$. Hence $\widetilde{E}_{\beta 1}$ is a relative divisor over $\widetilde{D}_{\beta}$ by Lemma 4 . Let $\tau_{\beta}: \widetilde{D}_{\beta} \rightarrow$ Div $X$ be an associated universal morphism. Let $\widetilde{D}_{\alpha}$ be the irreducible component of Div $X$ which contains $\tau_{\beta}\left(\widetilde{D}_{\beta}\right)$. As we have already remarked, actually we have $\tilde{D}_{\alpha} \subseteq^{\prime} \tilde{D}$ iv $X$. Moreover, $\tilde{D}_{\alpha}$ is non-isolated since $\widetilde{E}_{\beta 1, d}$ moves as well as $\widetilde{Z}_{\beta, d}$ when $d$ moves in $\widetilde{D}_{\beta}$, and it is non-transversal to $f$ since for any $d \in \widetilde{D}_{\beta}, \tilde{Z}_{\alpha, \tau_{\beta}(d)}$ $=\widetilde{E}_{\beta 1, d}$ and $f\left(\widetilde{E}_{\beta 1, d}\right)=\widetilde{Z}_{\beta, d} \neq Y$. We set $a\left(\tilde{D}_{\beta}\right)=\tilde{D}_{\alpha}$.

Conversely, let $\tilde{D}_{\alpha}$ be any irreducible component of 'D̃iv $X$ which is nonisolated and non-transversal to $f$. Let $F_{\alpha}:=\left(i d_{\tilde{D}_{\alpha}} \times f\right): \widetilde{D}_{\alpha} \times X \rightarrow \widetilde{D}_{\alpha} \times Y$. We set $\bar{Z}_{\alpha}:=F_{\alpha}\left(\tilde{Z}_{\alpha}\right) \subseteq \widetilde{D}_{\alpha} \times Y$. Then by Lemma $4, \bar{Z}_{\alpha}$ is a relative divisor over $Y$ since $\tilde{D}_{\alpha}$ is not transversal to $f$. Let $\tau_{\alpha}: \widetilde{D}_{\alpha} \rightarrow \tilde{D}$ iv $Y$ be an associated universal morphism. Let $\tilde{D}_{\beta}$ be the irreducible component which contains $\tau_{\alpha}\left(\widetilde{D}_{\alpha}\right)$. Then by the same argument as above $\widetilde{D}_{\beta} \subseteq \tilde{D}$ iv $Y$ and it is non-isolated (cf. Remark 1). Then we set $b\left(\widetilde{D}_{\alpha}\right)=\widetilde{D}_{\beta}$.

We now show that the above correspondences $a$ and $b$ are in fact bijective, inverse to each other, and have the property of the proposition. First, we note that from our construction it follows readily that $\tau_{\beta}$ is generically injective and moreover that each fiber of $\tau_{\alpha}$ is discrete; for any $d \in \tau_{\alpha}\left(\widetilde{D}_{\alpha}\right)$, the support of $\widetilde{Z}_{\alpha, d^{\prime}}$ is contained in $f^{-1}\left(\bar{Z}_{\alpha, d}\right)$ for each $d^{\prime} \in \tau_{\alpha}^{-1}(d)$ and hence by Remark $1 \operatorname{dim} \tau_{\alpha}^{-1}(d)$ $=0$. We further show that $\bar{Z}_{\alpha, d}$ is reduced if $\widetilde{Z}_{\alpha, d}$ is reduced and if $\tilde{Z}_{\alpha, d} \cap X_{U}$ is dense in $\widetilde{Z}_{\alpha, d}$. In fact, since $f_{U}$ is a smooth fiber space, we have $\widetilde{F}_{\alpha}^{-1}\left(\bar{Z}_{\alpha} \cap\left(\widetilde{D}_{\alpha} \times U\right)\right)$ $=\tilde{Z}_{\alpha} \cap\left(\tilde{D}_{\alpha} \times X_{U}\right)$, both sides being reduced. Hence $f^{-1}\left(\bar{Z}_{\alpha, d} \cap U\right)=\tilde{Z}_{\alpha, d} \cap X_{U}$, so that $\bar{Z}_{\alpha, d} \cap U$ is reduced if so is $\tilde{Z}_{\alpha, d}$. If further, $Z_{\alpha, d} \cap X_{U}$ is dense in $Z_{\alpha, d}$ then $\bar{Z}_{\alpha, d} \cap U$ also is dense in $\bar{Z}_{\alpha, d}$ and hence $\bar{Z}_{\alpha, d}$ also is reduced.

Now we fix $\widetilde{D}_{\beta} \cong{ }^{\prime}$ Div $X$. We consider the corresponding $\tilde{D}_{\alpha}=a\left(\widetilde{D}_{\beta}\right), \tau_{\beta}$ : 
$\tilde{D}_{\beta} \rightarrow \tilde{D}_{\alpha}$ and $\tau_{\alpha}: \tilde{D}_{\alpha} \rightarrow$ 'Div $Y$. Suppose that we have shown that $\tau_{\alpha} \cdot \tau_{\beta}=i d_{\tilde{D}_{\beta}}$ so that in particular $\tau_{\alpha}\left(\widetilde{D}_{\alpha}\right) \supseteqq D_{\beta}$. Then, since $\widetilde{D}_{\alpha}$ and $\widetilde{D}_{\beta}$ are normal and irreducible, $\tau_{\alpha}\left(\widetilde{D}_{\alpha}\right)=\tilde{D}_{\beta}$ and, in view of the generic injectivity of $\tau_{\beta}$ and the fact that $\operatorname{dim} \tau_{\alpha}^{-1}(d)$ $=0$, this would imply that $\tau_{\alpha}$ and $\tau_{\beta}$ give isomorphisms of $\widetilde{D}_{\alpha}$ and $\tilde{D}_{\beta}$ and that $b a$ =identity. So we show that $\tau_{\alpha} \cdot \tau_{\beta}=i d_{\tilde{D}_{\beta}}$. Let $V \subset \tilde{D}_{\beta}$ be a Zariski open subset such that $\tilde{Z}_{\beta, d}$ are reduced and $\tilde{Z}_{\beta, d} \cap U$ is dense in $\tilde{Z}_{\beta, d}$ for all $d \in V$. Then we have only to show that $\tau_{\alpha}\left(\left.\tau_{\beta}\right|_{V}\right)=i d_{V}$. This follows if we show that $\tilde{Z}_{\beta, d}=\bar{Z}_{\alpha, d^{\prime}}$ for any $d \in V$, with $d^{\prime}=\tau_{\beta}(d)$, as a subspace of $Y$. By our construction it is clear that $\widetilde{Z}_{\beta, d}=\left(\bar{Z}_{\alpha, d^{\prime}}\right)_{\mathrm{red}}$, while by what we have proved above $\bar{Z}_{\alpha, d^{\prime}}$ is reduced since $\tilde{Z}_{\alpha, d^{\prime}}=\widetilde{E}_{\beta 1, d}$ is reduced and $\left(\widetilde{E}_{\beta 1, d} \cap X_{U}\right)$ is dense in $\widetilde{E}_{\beta 1, d}$. Hence the assertion is proved.

Next we fix $\widetilde{D}_{\alpha}$. We consider the corresponding $\widetilde{D}_{\beta}=b\left(\widetilde{D}_{\alpha}\right), \tau_{\alpha}: \widetilde{D}_{\alpha} \rightarrow \widetilde{D}_{\beta}$, and $\tau_{\beta}: \widetilde{D}_{\beta} \rightarrow \tilde{D}$ iv $X$. Then just as above we show that $\tau_{\beta} \cdot \tau_{\alpha}=i d_{\tilde{D}_{\alpha}}$ and then that $a b=$ identity. We set $\varphi_{\beta \alpha}=\tau_{\beta}: \widetilde{D}_{\beta} \cong \tilde{D}_{\alpha}$.

It remains to show the existence of $d \in \operatorname{Div} X$ satisfying $\tilde{\mu}_{\alpha} \varphi_{\alpha \beta}=\bar{d}^{*} f^{*} \tilde{\mu}_{\beta}$. Write $\widetilde{E}=\widetilde{E}_{\beta 1} \cup \widetilde{E}_{\beta 2}$ for a unique relative divisor $\widetilde{E}_{\beta 2}$ over $\widetilde{D}_{\beta}$ with $\widetilde{E}_{\beta 1} \nsubseteq \widetilde{E}_{\beta 2}$. Then by our definition of $\widetilde{E}_{\beta 1}$ we have $\widetilde{E}_{\beta 1} \cap\left(\widetilde{D}_{\beta} \times X_{U}\right)=\widetilde{E}_{\beta} \times X_{U}$. Hence if $A:=X-X_{U}$, then $\widetilde{E}_{\beta 2} \subseteq \widetilde{D}_{\beta} \times A$. Hence by Remark $1 \widetilde{E}_{\beta 2} \rightarrow \widetilde{D}_{\beta}$ is a constant family, so that the image of an associated universal morphism $\tau_{\beta 2}: \tilde{D}_{\beta} \rightarrow \tilde{D}$ iv $X$ is a unique point. Then it suffices to take this point as $d$. (When $\widetilde{E}_{\beta 2}=\varnothing$, we set $d=0$.)

q.e.d.

Proof of Porposition. Let $D_{\alpha}=D_{\gamma, \alpha}$ be any irreducible component of $D_{\gamma, \text { red }}$ and $\bar{D}_{\alpha}=\mu_{\gamma}\left(D_{\alpha}\right) \subseteq \operatorname{Pic}_{\gamma} X$. Then it suffices to show that $\bar{D}_{\alpha}$ is contained in some orbit $P_{\gamma}^{a}=P_{\gamma}^{a}(\alpha)$. In fact, if $\bar{D}_{\gamma}^{i}$ is any connected component of $\bar{D}_{\gamma, \mathrm{red}}$ and $\bar{D}_{\gamma}^{i}=\bigcup_{\alpha \in \mathscr{O U}_{i}} \bar{D}_{\alpha}$, then $\bigcup_{\alpha \in \mathscr{I T}_{i}} P_{\gamma}(\alpha)$ also is connected and hence $P_{\gamma}(\alpha)=P_{\gamma}\left(\alpha^{\prime}\right)$ for any $\alpha, \alpha^{\prime} \in \mathfrak{U}_{i}$ since the orbits are mutually disjoint. Hence $\bar{D}_{\gamma}^{i} \subseteq P_{\gamma}^{a}$ for a unique orbit $P_{\gamma}^{a}$. Now we show that $\bar{D}_{\alpha} \subseteq P_{\gamma}^{a}$ for some $P_{\gamma}^{a}$. First, by Lemma 5 we infer that we may assume that $\widetilde{D}_{\alpha} \subseteq{ }^{\prime}$ Div $X$. If $\tilde{D}_{\alpha}$ is isolated, then the assertion is clearly true. So we may assume that $\tilde{D}_{\alpha}$ is not isolated. We take a holomorphic model (*) of algebraic reduction of $X$. Then by Lemma 7 applied to $\varphi$, we can replace $X$ by $X^{*}$ so that we may assume from the beginning that $X=X^{*}$ and $f$ is defined on $X$. Now by Fischer-Forster [2] if $\tilde{D}_{\alpha}$ is transversal to $f$, then $D_{\alpha}$ is isolated (cf. Remark 1). Hence we may further assume that $\tilde{D}_{\alpha}$ is not transversal. Then applying Lemma 7 this time to $f$, Proposition follows immediately. 


\section{§3. Proof of the Theorems}

Let $X$ be a normal compact complex space. Let $r: \tilde{X} \rightarrow X$ be a resolution. Since $X$ is normal so that $r_{*} \mathcal{O}_{\tilde{X}} \cong \mathcal{O}_{X}$, the natural morphism $r^{*}: \operatorname{Pic} X \rightarrow \operatorname{Pic} \tilde{X}$ is injective.

Lemma 8. For each $\gamma \in N S(X), r^{*}\left(\operatorname{Pic}_{\gamma} X\right)$ is a closed submanifold in Pic $X$. In particular $r^{*}$ is a closed embedding.

Proof. It suffices to show that $r^{*}\left(\operatorname{Pic}_{0} X\right)$ is closed in $\operatorname{Pic}_{0} \tilde{X}$. Consider the following commutative diagram of exact sequences

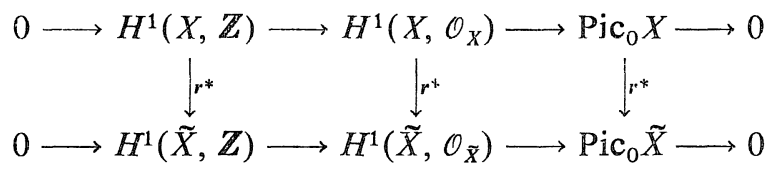

where the vertical maps are injective and the horizontal sequences come from the exponential sequences on $X$ and $\tilde{X}$. Then it is enough to show that the subgroup in $H^{1}\left(\tilde{X}, \mathcal{O}_{\tilde{X}}\right)$ generated by $H^{1}(\tilde{X}, \mathbb{Z})$ and $H^{1}\left(X, \mathcal{O}_{X}\right)$ is closed. Fisrt, recall that we have the natural inclusions $H^{1}\left(X, \mathbb{R}^{2}\right) \rightarrow H^{1}\left(X, \mathcal{O}_{X}\right)$ and $H^{1}(\tilde{X}, \mathbb{R}) \rightarrow H^{1}\left(\tilde{X}, \mathcal{O}_{\tilde{X}}\right)$ of real vector spaces (cf. [6] IX, Prop. 3.2). Then, clearly the subgroup in $H^{1}(\tilde{X}, \mathbb{R})$ generated by $H^{1}(X, \mathbb{R})$ and $H^{1}(\tilde{X}, \mathbb{Z})$ is closed. Since $H^{1}\left(X, \mathcal{O}_{X}\right)$ is a vector subspace of $H^{1}\left(\tilde{X}, \mathcal{O}_{\tilde{X}}\right)$, from this the lemma follows immediately.

q.e.d.

Proof of Theorem 1. Clearly we may assume that $X$ is connected. Suppose that $X$ is nonsingular. Then, as we have already noted, Theorem follows immediately from Lemma 3 and Proposition. So suppose that $X$ is not nonsingular. Let $\gamma \in N S(X)$ be arbitrary. Then we have only to show that any connecled component $\bar{D}_{\gamma, \alpha}$ of $\bar{D}_{\gamma}$ is projective (cf. $\S 1$ ). Let $r: \tilde{X} \rightarrow X$ be a resolution of $X$. Then there exists a unique $\tilde{\gamma} \in N S(\tilde{X})$ such that $r^{*}\left(\operatorname{Pic}_{\gamma} X\right)$ $\subseteq \operatorname{Pic}_{\tilde{\gamma}} \tilde{X}$. Then by Lemma 1 and the definition of $r^{*}$ we have $r^{*}\left(\bar{D}_{\gamma, \text { red }}\right)$ $=\bar{D}_{\tilde{\gamma}, \text { red }} \cap r^{*}\left(\operatorname{Pic}_{\gamma} X\right)$. On the other hand, every connected component of $\bar{D}_{\tilde{\gamma}, \text { red }}$ is contained in some orbit $P_{\tilde{\gamma}}^{a}$ of $P^{a}=P^{a}(\tilde{X})$ in $\operatorname{Pic}_{\tilde{\gamma}} \tilde{X}$ by Proposition. Hence by Lemma $8, r^{*}\left(\bar{D}_{\gamma, \alpha, \mathrm{red}}\right)$ is a closed analytic subspace of $P_{\tilde{\gamma}}^{a}$. Therefore noting that $r^{*}$ is an embedding, by Lemma $3, \bar{D}_{\gamma, \alpha}$ is projective as was desired.

Proof of Theorem 2. Since $D_{\alpha}(A)=\rho\left(Z(X) \cap\left(D_{\alpha} \times A\right)\right), D_{\alpha}(A)$ is analytic. 
On the other hand, we have $D_{\alpha}(A)=\cup_{x \in A} D_{\alpha}(x)$ where $D_{\alpha}(x)=D_{\alpha}(\{x\})$. Now $D_{\alpha}(x)=Z(X) \cap\left(D_{\alpha} \times\{x\}\right) \subseteq D_{\alpha}$ is regarded naturally as a divisor on $D_{\alpha}$ (not necessarily reduced). It then follows that there exist a finite number of points $x_{1}, \ldots$, $x_{m} \in X$ such that $D_{\alpha}(A)=\bigcup_{i=1}^{m} D_{\alpha}\left(x_{i}\right)$ as a set. (Recall that $D_{\alpha}$ is compact by Theorem 1.) Thus it suffices to show that $D_{\alpha}\left(x_{i}\right)$ is ample for any $i$. We first note that under our assumption there exists a Zariski open subset $U$ of $X$ containing $x_{\imath}$ such that $D_{\alpha}(x)$ is a divisor on $D_{\alpha}$ for any $x \in U$. Further since the divisors $D_{\alpha}(x), x \in U$, are all mutually algebraically equivalent, it suffices to show that $D_{\alpha}\left(x_{0}\right)$ is ample for some fixed $x_{0} \in U$.

Claim. For any nowhere discrete reduced analytic subspace $B$ of $D_{\alpha}$ we can find $x \in U$ such that 1) $D_{\alpha}(x)$ intersect each irreducible component of $B$ and 2) $B \cap D_{\alpha}(x)$ is nowhere dense in $B$.

In fact, let $B_{i}, i=1, \ldots, r$, be the irreducible components of $B$. Fix a point $b_{i}$ $\in B_{i}-\cup_{j \neq i} B_{j}$ for each $i$. Let $Z_{b_{i}} \subseteq X$ be the corresponding divisor and then take any $x \in U-\bigcup_{i=1}^{r} Z_{b_{i}}$. Then obviously $b_{i} \notin D_{\alpha}(x)$. Hence 2$)$ is satisfied. Moreover since the natural map $Z_{B_{i}} \rightarrow X$ is surjective by our assumption that $\operatorname{dim} B_{i}$ $>0, D_{\alpha}(x) \ni b_{i}^{\prime}$ for some $b_{i}^{\prime} \in B_{i}$. Hence 1) also is true. The claim is proved.

Now using this claim inductively we see that for any $p \geqq 0$ and any complex subvariety $C$ of dimension $p$ of $D_{\alpha}$, we can always find $x_{1}, \ldots, x_{p} \in U$ such that $D_{\alpha}\left(x_{1}\right) \cap \cdots \cap D_{\alpha}\left(x_{p}\right) \cap C$ is a nonempty finite set of points. This implies that the intersection number $D_{\alpha}\left(x_{0}\right)^{p} \cdot C=D_{\alpha}\left(x_{1}\right) \cdot D_{\alpha}\left(x_{2}\right) \cdots \cdot D_{\alpha}\left(x_{p}\right) \cdot C>0$ (cf. [7]). Hence by Nakai crieterion (cf. [7]) $D_{\alpha}\left(x_{0}\right)$ is ample.

q.e.d.

Remark 2. The above proof shows in fact the following: For any complex variety $X$ and any compact subspace $B$ of $\operatorname{Div} X, B(A)=\left\{d \in B ; Z_{X, d} \cap A\right.$ $\neq \varnothing\}$ is a support of an ample divisor if it is a divisor on $B$ at all. (Note that $B(A)=\cup_{x \in A} B(x)$ and $B(x)$ has the natural structure of a locally principal analytic subspace of B.) See Banica, C., and Ueno, K., J. Math. Kyoto Univ., 20 (1980), 381-389, for the intersection theory on a general compact complex space generalizing that of [7]. Further $B-B(A)$ is affine if $B(A)$ contains no irreducible component of $B_{\text {red. }}$. The result of Ohsawa [8] mentioned in the introduction implies that $B-B(A)$ is Stein. 


\section{References}

[0] Campana, F., Sur les sous espaces maximaux d'un espace analytique compact, preprint.

[1] Douady, A., Le problème de modules pour les sous-espaces analytiques complexes d'un espace analytique donné, Ann. Inst. Fourier, Grenoble, 16 (1966), 1-95.

[2] Fischer, W. and Forster, O., Ein Endlichkeitssatz für Hyperflächen auf kompakten komplexen Räumen, J. Reine Angew. Math., 306 (1979), 88-93.

[3] Fujiki, A., Relative algebraic reduction and relative Albanese map for a fiber space in $\mathscr{C}$, Publ. RIMS, Kyoto Univ., 19 (1983), in press.

[4] Grothendeick, A., Fondements de la géométrie algébrique (Extraits du Seminaire Bourbaki 1957-1962), Paris, 1962.

[ 5 ] — - Elements de géométrie algébrique IV, Publ. Math. I. H. E. S., 32 (1967).

[6] - Technique de construction en géométrie analytique, 13ème année, Seminaire H. Cartan, 1960/61.

[ 7 ] Kleiman, S., Toward a numerical criterion of ampleness, Ann. of Math., 84 (1966), 293-344.

[ 8 ] Ohsawa, T., Completeness of noncompact analytic spaces, preprint.

[ 9 ] Fujiki, A., Closedness of the Douady spaces of compact Kähler spaces, Publ. RIMS, Kyoto Univ., 14 (1978), 1-52. 
